\title{
Study of Outcomes and Characteristics of COVID-19 Positive Geriatric Patients in a Scottish Rehabilitation Community Hospital.
}

\section{Ying Ern Elena Ong ( $\square$ elena.ong@nhs.scot )}

Royal Victoria Hospital

\section{Zhong Wei Khor}

Ninewells Hospital

Ryan McGovern

Hairmyres Hospital

\section{Sean Testrow}

Raigmore Hospital

Carolyn Tipton

Ninewells Hospital

\section{Research Article}

Keywords: Positive Geriatric, Scottish Rehabilitation, demographic data

Posted Date: December 29th, 2020

DOI: https://doi.org/10.21203/rs.3.rs-125405/v1

License: (9) This work is licensed under a Creative Commons Attribution 4.0 International License. Read Full License 


\section{Abstract}

\section{Background}

A dedicated step-down unit was instated in a community geriatric rehabilitation hospital in Dundee to meet the ongoing rehabilitation needs of a Scottish COVID-19 positive geriatric patient cohort. As the profound chronic health effects of COVID-19 on our ageing populations are still poorly understood, our study aims to investigate patient characteristics and outcomes in this cohort of patients stepped-down to the community geriatric hospital COVID-19 ward.

\section{Design}

This was a retrospective observational study of all patients admitted to the COVID-19 step-down unit. Patient demographic data and data on pre- and post-COVID-19 infection patient functional status, clinical frailty score, mobility status and mortality were collected to quantify extent of physical-functional decline in this patient cohort from inpatient notes and electronic databases.

Results

33 COVID-19 positive patients were included in this study. $36 \%$ of patients lost their independent function status and there was a $45 \%$ increase (15 patients) in care requirements, accompanied by a $41 \%$ decline mobility. Average length of inpatient stay of the surviving population was longer compared to the cohort who died, (35.3 days vs 20.6 days, $p=0.028$ ). Stroke as a co-morbidity was associated with increased mortality in this patient cohort $(p=0.006,<0.05)$. Obstructive lung disease is noted to be associated with an increased length of stay (33.4 days vs 20 days, $p=0.011$ ).

\section{Conclusions}

This study demonstrates quantifiable physical functional decline demonstrated by loss of independent function, increase in care requirements, increased frailty, and reduced mobility in this geriatric cohort post COVID-19 infection.

\section{Introduction}

Geriatric patients worldwide have paid a great physical and emotional toll in this global COVID-19 pandemic. Even after successfully surviving through the acute phase of a COVID-19 attack, they are left with undesirable knock-on effects on their health and wellbeing in the form of physical-functional decline and increased frailty. Many of these geriatric COVID-19 survivors are not suitable for direct hospital discharge as they require various levels of rehabilitation and multi-disciplinary team input to identify a new functional baseline to guide tailored care planning to facilitate a safer hospital discharge.

As the acute 'red zone' COVID-19 assessment ward was not ideal for rehabilitation and discharge planning activities, the establishment of an 'amber zone' COVID-19 clinical area was important to deliver 
medical/nursing care while facilitating rehabilitation services to COVID-19 positive geriatric patients, to maximise post-COVID recovery outcomes. This patient need led to the establishment of a designated COVID-19 ward within Royal Victoria Hospital, Dundee (community geriatric rehabilitation hospital).

Numerous recently published literature has highlighted the clinical features of COVID-19 infection in the geriatric patient population during acute phase infection." However there is a current paucity of research scrutinising outcomes in patients who survived the acute phase infection and were stepped-down to rehabilitation hospital settings for rehabilitation prior to hospital discharge.

The aims of this retrospective descriptive research were to study patient characteristics and outcomes in a cohort of COVID-19 positive geriatric patients admitted to a community geriatric hospital COVID-19 ward for rehabilitation, ward level medical treatment, and discharge planning. This study also specifically investigates the extent of physical functional decline and increased frailty experienced by this patient group following COVID-19 infection.

\section{Methods}

The authors were part of the medical team providing care within the research setting. We conducted a retrospective observational cohort study to investigate the inpatient progress and 30-day follow-up for all patients admitted to a COVID-19 rehabilitation ward within the hospital between 10/4/2020-19/6/2020 Primary outcome measures were change in mobility, change in care requirements, change in level of frailty measured using the Clinical Frailty Score, as well as overall survival and length of stay.

Caldicott Guardian approval was granted to allow data collection. All patients admitted to the COVID-19 rehabilitation ward had either been transferred from another non-COVID-19 area within the same hospital after testing positive for COVID-19 or were stepped down from the acute hospital COVID-19 areas for ongoing care and rehabilitation. A total of 33 patients were admitted to the ward during the study period.

Data relating to patient demographics (age and gender), clinical indicators (medical history, medications, and biochemistry/ haematology blood test results at time of diagnosis), baseline level of function (preCOVID-19 mobility, existing care requirements, and level of frailty) were collected retrospectively from the medical clerking documents, electronic medical records, and clinical results portal by members of the research team. Post-COVID-19 data regarding inpatient mortality rate, changes in mobility, care requirements and clinical frailty (with the score being assigned at time of discharge or death by a medical practitioner actively involved in the care of the patient) was also collected.

Data was stored in an encrypted spreadsheet document accessible only to the research team. Univariate analysis using SPSS (version 21, IBM) was performed on each patient factor against two outcomes: length of hospital stay; and mortality.

\section{Results}


There was an equal distribution of gender in this cohort of 33 patients ( $51.5 \%$ male/ $48.5 \%$ female), with the mean age at presentation being 85 years (Range: 71-99 years old). Local outbreaks from this community rehabilitation hospital's wards contributed more than half (54\%) of this COVID-19 ward admission rates. The remainder were stepped down from the secondary care center's (Ninewells Hospital) acute COVID-19 admission unit.

$100 \%$ of this study cohort were multi-morbid as per NICE guidelines definition of multi-morbidity, with stroke being the most common comorbidity (58\%) [Table 1]. In the same way, polypharmacy is present in $100 \%$ of the study cohort, this is based on the definition of being on 2 or more medications. 
Table 1

Patient Demographics

\begin{tabular}{|ll|}
\hline Patient Demographics & N $=33$ \\
\hline Number of patients & $85(71-99)$ \\
\hline Mean Age in Years (Range) & \\
\hline Gender & $17(52 \%)$ \\
\hline Male & $16(48 \%)$ \\
\hline Female & \\
\hline Admission Source & $15(46 \%)$ \\
\hline Transfer from Acute CoVID admission ward & $18(54 \%)$ \\
\hline Own Rehab Hospital patients & Present $(\%)$ \\
\hline Medical Co-morbidities & $19(58 \%)$ \\
\hline Stroke & $16(48 \%)$ \\
\hline Hypertension & $13(39 \%)$ \\
\hline Ischaemic heart disease & $12(36 \%)$ \\
\hline Type Il diabetes mellitus & $12(36 \%)$ \\
\hline Chronic kidney disease & $9(27 \%)$ \\
\hline Atrial fibrillation & $9(27 \%)$ \\
\hline Heart failure & $6(24 \%)$ \\
\hline Obstructive lung disease & Present (\%) \\
\hline Medication & $5(15 \%)$ \\
\hline Ace inhibitor & $2(6 \%)$ \\
\hline Alpha receptor blocker & $10(30 \%)$ \\
\hline Diuretic & $16(49 \%)$ \\
\hline Beta blocker & $15(45 \%)$ \\
\hline Statin & \\
\hline Antiplatelet & \\
\hline Anticoagulation & $12 \%)$ \\
\hline Symptoms at Detection & \\
\hline
\end{tabular}




\begin{tabular}{|ll|}
\hline Patient Demographics & \\
\hline Fever only & $3(9 \%)$ \\
\hline Respiratory symptoms only & $7(21 \%)$ \\
\hline Fever and respiratory symptoms both & $18(55 \%)$ \\
\hline Atypical & $1(3 \%)$ \\
\hline
\end{tabular}

Biochemically, most patients presented with lymphopenia (72.7\%) and an elevated CRP (87.9\%). However, leucocyte count remained largely unaffected $(66.7 \%)$ with normal neutrophil and platelet counts in this patient cohort. Approximately half (55\%) of the patients had both pyrexia and respiratory symptoms at COVID-19 detection. Notably, $12 \%$ of positive swabs came from completely asymptomatic patients with 1 patient presenting atypically with delirium as his only feature. 
Table 2

Patient outcomes Pre- and Post-COVID-19.

\begin{tabular}{|c|c|c|}
\hline & Pre COVID-19 (\%) & Post COVID-19 (\%) \\
\hline \multicolumn{3}{|l|}{ Function Status } \\
\hline Independent & $13(39 \%)$ & $1(3 \%)$ \\
\hline Home with Package of Care & $17(52 \%)$ & $17(52 \%)$ \\
\hline Sheltered housing & $2(6 \%)$ & 0 \\
\hline Care home & $1(3 \%)$ & $4(12 \%)$ \\
\hline Death & 0 & $11(33 \%)$ \\
\hline \multicolumn{3}{|l|}{ Clinical Frailty Score (Dalhousie) } \\
\hline 3 & $4(12 \%)$ & $1(3 \%)$ \\
\hline 4 & $8(25 \%)$ & $1(3 \%)$ \\
\hline 5 & $10(30 \%)$ & $5(15 \%)$ \\
\hline 6 & $10(30 \%)$ & $12(37 \%)$ \\
\hline 7 & $1(3 \%)$ & $2(6 \%)$ \\
\hline 8 & 0 & $1(3 \%)$ \\
\hline Death & 0 & $11(33 \%)$ \\
\hline \multicolumn{3}{|l|}{ Mobility status } \\
\hline Independent & $12(36 \%)$ & $1(3 \%)$ \\
\hline Walking aid (ZF, stick, triwheeler, wheelchair) & $21(64 \%)$ & $21(64 \%)$ \\
\hline Death & 0 & $11(33 \%)$ \\
\hline \multicolumn{3}{|l|}{ Mortality } \\
\hline Dead & 0 & $11(33 \%)$ \\
\hline Alive & $33(100 \%)$ & $22(69 \%)$ \\
\hline
\end{tabular}

A significant deterioration in the function status of our patient cohort has been observed. This is defined by either an introduction of new package of care where there was previously none, an increase of existing package of care or a new requirement of institutional care. $36 \%$ of patients' loss their independent function status post COVID- 19 , this is accompanied by a $45 \%$ increase (15 patients) in care requirements [Table 2]. 
There has also been a decline (41\%) in mobility seen in this cohort post COVID-19 which is defined as a new requirement of walking aids such as Zimmer/Gutter frames when patients were previously independently mobile without aid. Similarly, an increase in frailty (14 patients, $64 \%$ ) has been observed in this cohort as defined by an increase in their Dalhousie clinical frailty score (Fig. 1).

\section{Patient outcomes}

11 patients (33\%) unfortunately passed away due to COVID-19 related disease or its complication. Univariate analysis has revealed no statistically significant relationship between gender, age of presentation, use of ace inhibitors, clinical frailty score at present with length of stay or survival. However, stroke as a comorbidity was associated with increased mortality and shorter overall survival in this patient cohort ( 49.7 days vs 66.3 days, $p=0.048,<0.05$, Appendix 1 ). Additionally, obstructive lung disease is noted to be associated with an increased length of stay (33.4 days vs 20 days, $p=0.011$, Appendix 2).

The average length of stay of the surviving population is longer compared to the dead population and this is statistically significant ( 35.3 days vs 20.6 days, $p=0.028$ ). Of the surviving group, 8 patients $(36 \%)$ were readmitted within 30 days of discharge, with 3 patients who unfortunately passed away within 30 days of discharge.

\section{Discussion}

The initial COVID-19 infection control response during the first inpatient outbreaks in this community geriatric rehabilitation hospital (Royal Victoria Hospital) was to transfer PCR-positive patients across to the main secondary care hospital (Ninewells Hospital) for cohorting.

As outbreak numbers increased across both sites, it was necessary to establish a COVID-19 ward within the rehabilitation geriatric hospital. The rationale for this was multiple-fold: to reduce burden on ambulance services to transfer a growing number of patients across sites, alleviate rapidly expanding bed pressures for COVID-19 positive admissions from the community, as well as to reduce patient distress from rapid transfers to unfamiliar clinical environments.

Other changes made by the multidisciplinary team at RVH included reducing the number of beds per bay to allow for adequate $2 \mathrm{~m}$ social distancing, and the delivery of rehabilitation activities within the COVID19 ward itself as well as utilising separate rehabilitation equipment to reduce risk of patient-to-patient transmission in the shared gym.

Patients admitted to this COVID-19 rehabilitation ward were PCR positive on admission and underwent regular surveillance COVID-19 testing. When they were found to be COVID-19 negative, the patient was transferred to a non-COVID-19 rehabilitation ward to carry on rehabilitation and discharge planning. While there were varying policies in test of cures during 3 months of the outbreak, $x 2$ negative swabs was mandatory for all care home discharges due to serious clinical risk posed to the existing residents. 
Patients who have reached their full rehabilitation potential and were fit for discharge home but remained COVID-19 positive on PCR could be discharged directly from the COVID-19 ward with self-isolating instructions for 14 days. In such cases, family and carers were informed about the patient's COVID-19 status prior to discharge for appropriate precaution taking.

More than half of patients admitted to this COVID-19 rehabilitation ward were from various COVID-19 outbreaks onsite, which suggests that geriatric patients within a rehabilitation hospital setting are at significant risk of COVID-19 infection. Increased care requirements by this patient demographic often necessitate allied health professionals and ward staff to be in proximity with patients to assist with activities of daily living. This is challenging for effective social distancing to occur in such clinical environments, which perpetuates risk of infection spread despite diligent use of face masks and PPE.

It is interesting to note that a significant portion of patients who were COVID-19 positive on PCR were asymptomatic at detection. This suggests that a high clinical suspicion for COVID-19 should remain as patients can be widely asymptomatic even when positive. Blood result trends such as lymphopenia and raised CRP seen in this patient cohort was in line with current literature. Unfortunately, there was inconsistent requesting of coagulation profiles at presentation for accurate data collection and meaningful comparison with current literature about COVID-19 coagulopathy.

Ward staff had noticed that stroke patients in this cohort who developed COVID-19 suffered a higher mortality rate and length of hospital stay, and this was statistically significant on univariate analysis. A suggested explanation to this is that patients who have stroke often present with other organ system comorbidities contributing to overall pre-COVID-19 frailty and increased rehabilitation requirements. A prolonged hospital stay could also predispose these patients to prolonged exposure risks to hospital acquired infections resulting increased mortality.

It is clear from current literature that COVID-19 disproportionally affects older patients due to diminished immune function and multi-morbidities. This patient group is most likely to require hospital admission, and most likely to die from COVID-19 infection. Significant physical functional decline is a recognised outcome in the surviving geriatric COVID-19 population and our study demonstrated this though quantifiable deterioration in mobility, increase in care status and increased frailty post-COVID infection. This supports our theory that COVID-19 survivors require more time to rehabilitate to a new functional baseline safe for hospital discharge, as demonstrated by the statistically significant increased length of hospital stay in our surviving cohort. This further reiterates the importance of a COVID-19 rehabilitation unit inpatient service for COVID-19 positive patients to engage in rehabilitation activities as tolerated while remaining PCR positive, to maximise recovery.

In this study, we observed an increase in Clinical Frailty Scores (CFS) in the surviving population post COVID-19 infection. This data was collected retrospectively, however there is potential for rehabilitation hospitals to utilise the CFS assessment in conjunction with the Comprehensive Geriatric Assessment (CGAs) patient co-morbidities at point of admission as tools to rationalise treatment goals and patient outcomes. 
Such use of CFS in patient care in COVID-19 has been recommended by NICE Guidelines to guide clinical decision making, for example, the NICE critical care algorithm suggests that COVID-19 positive patients with a CFS $\geq 5$ would not benefit from admission to ICU. Our paper did not find a statistical significance in survival rates in patients with a lower clinical frailty score at COVID-19 presentation, which suggests that CFS should not be used in isolation to guide clinical decision making. Instead, as Hubbard et al suggested, the CFS should be considered as an important component of a holistic patient-centred approach to assessment taking into consideration biomedical factors such as existing co-morbidities and severity of acute COVID-19 infection.

A small sample size of such a unique patient cohort described in our study makes it difficult to accurately extrapolate data for application to the wider Scottish population. Strong multi-disciplinary team involvement (physiotherapy, occupational therapy, social work, nursing) should be considered in the early stages of COVID-19 research planning to adopt a holistic problem-solving approach towards the various challenges in COVID-19 rehabilitation.

Although not directly addressed through this study, it is important to explore the emotional and mental health challenges faced by geriatric patients through prolonged COVID-19 related hospital admission. One of the unique struggles of the COVID-19 patient journey is the emotional challenges of prolonged social isolation through stringent "restricted visitation" hospital infection control policies. This highlights the complexities in balancing public health concerns of pandemic control, while remaining patientcentred by ongoing reflection on how we can improve patient journeys for our population's most vulnerable.

\section{Conclusion}

This study presents a care model of an onsite COVID-19 ward in a geriatric rehabilitation hospital and demonstrates patient outcomes such as quantifiable physical functional decline and increased frailty in the geriatric cohort post COVID-19 infection. Stroke as a comorbidity was associated with increased mortality, whereas obstructive lung disease was associated with a prolonged length of hospital stay in this cohort. The mortality rate of this cohort is $33 \%$ and the surviving population require a longer length of stay to be well enough for discharge. Geriatric community rehabilitation hospitals are highly therapy and personal care intensive, possibly contributing to difficulties in consistent social distancing practices for adequate infection control. Further innovative measures are required for outbreak risk reduction in such clinical environments.

\section{Declarations}

- Ethics approval and consent to participate

- This study has been granted Caldicott guardianship as per local health board information governance standards. All data collection and utilisation were executed in adherence to 
Caldicott principles. Informed consent was obtained from all study subjects for anonymised data to be collected.

- Consent for publication

-Not applicable

- Availability of data and materials

- Not applicable

- Competing interests

-None

- Funding

-None

- Authors' contributions

-EO designed and lead the study. She coordinated the data gathering process and contributed to the writing of the manuscript.

-ZK assisted with the writing of the manuscript text as well as data analysis for the study.

-RM assisted with data gathering and contributed to the writing of the study's methodology.

-ST assisted with data gathering and contributed to the writing of the study's introduction.

-CT acted as the study supervisor and provided direction for publication.

- Acknowledgments

We would like to acknowledge the hard work and resilience of the RVH multi-disciplinary team comprising of nursing staff, physiotherapists, occupational therapists, social workers, pharmacists, infection control nurses, doctors and domestic staff who have worked hard to deliver the best possible care to these patients despite extremely challenging circumstances.

\section{References}

[1] Miles A, Webb TE, Mcloughlin BC, et al. Outcomes from COVID-19 across the range of frailty: excess mortality in fitter older people. Eur Geriatr Med. 2020;11(5):851-855. doi:10.1007/s41999-020-00354-7

[2] Bianchetti A, Bellelli G, Guerini F, et al. Improving the care of older patients during the COVID-19 pandemic. Aging Clin Exp Res. 2020;32(9):1883-1888. doi:10.1007/s40520-020-01641-w 
[3] Lithander FE, Neumann S, Tenison E, et al. COVID-19 in older people: a rapid clinical review. Age Ageing. 2020;49(4):501-515. doi:10.1093/ageing/afaa093

[4] Perrotta F, Corbi G, Mazzeo G, et al. COVID-19 and the elderly: insights into pathogenesis and clinical decision-making [published correction appears in Aging Clin Exp Res. 2020 Sep;32(9):1909]. Aging Clin Exp Res. 2020;32(8):1599-1608. doi:10.1007/s40520-020-01631-y

[5] Nanda A, Vura NVRK, Gravenstein S. COVID-19 in older adults. Aging Clin Exp Res. 2020;32(7):11991202. doi:10.1007/s40520-020-01581-5

[6] National Institute for Health and Care Excellence. Multimorbidity: clinical assessment and management. https://www.nice.org.uk/guidance/ng56/evidence/full-guideline-pdf-2615543103 (accessed 22 September 2020).

[7] Masnoon N, Shakib S, Kalisch-Ellett L, Caughey GE. What is polypharmacy? A systematic review of definitions. BMC Geriatr. 2017;17(1):230. Published 2017 Oct 10. doi:10.1186/s12877-017-0621-2

[8] Rockwood K, Song X, Macknight C, Bergman H, Hogan DB, McDowell I, Mitnitski A. A global clinical measure of fitness and frailty in elderly people. CMAJ. 2005 Aug 30;173(5):489-95

[9] Centres for Disease Control and Prevention. Interim Guidance on Management of Coronavirus Disease 2019 (COVID-19) in Correctional and Detention Facilities. https://www.cdc.gov/coronavirus/2019ncov/community/correction-detention/guidance-correctional-detention.html (accessed 22 November 2020).

[10] Pourbagheri-Sigaroodi A, Bashash D, Fateh F, Abolghasemi H. Laboratory findings in COVID-19 diagnosis and prognosis. Clin Chim Acta. 2020;510:475-482. doi:10.1016/j.cca.2020.08.019

[11] Chinnadurai R, Ogedengbe $O$, Agarwal $P$, et al. Older age and frailty are the chief predictors of mortality in COVID-19 patients admitted to an acute medical unit in a secondary care setting- a cohort study. BMC Geriatr. 2020;20(1):409. Published 2020 Oct 16. doi:10.1186/s12877-020-01803-5

[12] National Institute of Clinical Excellence. COVID-19 rapid guideline: critical care in adults. https://www.nice.org.uk/guidance/ng159 (accessed 22 September 2020).

[13] Hubbard RE, Maier AB, Hilmer SN, Naganathan V, Etherton-Beer C, Rockwood K. Frailty in the face of COVID-19. Age Ageing. 2020;49(4):499-500. doi:10.1093/ageing/afaa095

\section{Figures}




\section{Clinical Frailty Score Pre and Post COVID}

15

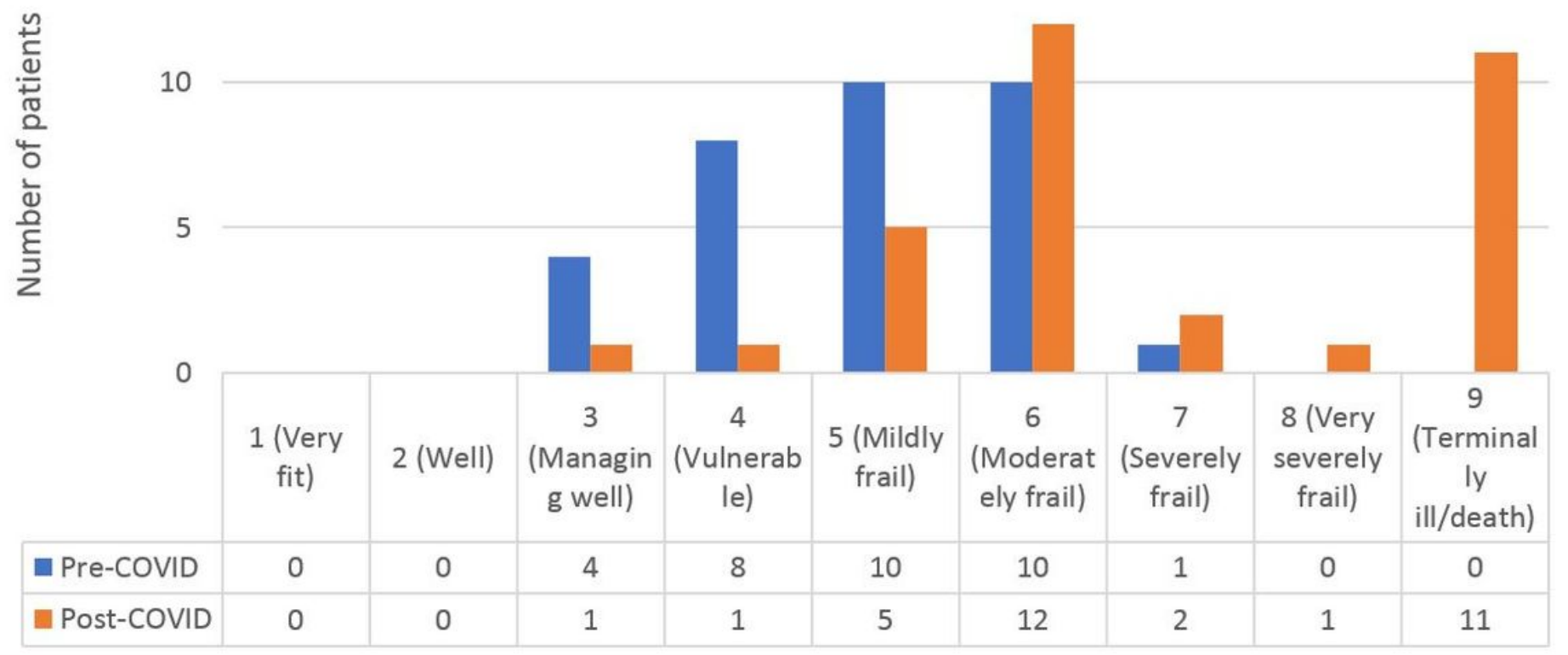

Figure 1

Clinical frailty score pre- and post-COVID.

\section{Supplementary Files}

This is a list of supplementary files associated with this preprint. Click to download.

- 5.Appendix.docx 